\title{
中枢八肽胆震收缩素促进大鼠失血 性休克的恢复
}

\author{
梅林韩济生
}

(北京医科大学生理教研室, 北京 100083)

\section{关镜调八肽胆㐮收缩索、失血性休克、平均动脉血压}

有资料表明, 中枢八肽胆衰收缩素 (CCK-8) 可对抗外源性注射阿片肽和由内源性阿 片肽介导 ${ }^{[2,3]}$ 的镇痛作用. 我们最近的工作表明, 阿片肽引起的降血压作用也能被中枢注 射 CCK-8 所翻转(梅林等,待发表). 上述实验结果都倾向于说明 CCK-8 有强烈的抗阿片肽作 用,可能是体内的抗阿片物质 ${ }^{[2,3]}$.

我室以前的工作表明, 失血性休克大鼠昘髉蛛网膜下腔 (ith.) 灌流液中甲啡肽和亮啡肽 样免度活性物质 (MEK-ir, LEK-ir) 的含量明显增加 ${ }^{[4]}$, ith 注射脑啡肽抗血清或阿片受体 拮抗剂能够促进休克后大鼠平均动脉血压 (MAP) 的恢复 ${ }^{\text {" }}$, 说明失血性休克时内源性阿片 系统的功能增强,对心血管活动起抑制作用.

如果 CCK-8 具有抗阿片肽作用,在失血性休克的病理条件下,体内 CCK-8 系统很可能 也被激活、释放增多, 从而与阿片肽的心血管抑制效应相抗衡，本文对这一设想进行了验证。

\section{一、材料与方法}

1. 动物和试剂用体重 300-350g 雄性 Wistar 大鼠,戊巴比妥钠 $(50 \mathrm{mg} / \mathrm{kg}$ 体重, 腹 腔内注射)麻醉下进行各项手术操作, 实验过程中以辐射热维持动物直肠温度 $38-38.5^{\circ} \mathrm{C}$.

CCK-8 为美国 Squibb 药厂生产, 丙谷胺为意大利 Rotta 实验室患赠, CCK-8 碩标物 （ㅍI-BH-CCK-8）为英国 Amersham 公司生产, CCK-8 抗血清为美国洪昭雄博士惠赠.

2. ith. 插管及注射 参照 $\mathrm{Yaksh}^{[6]}$ 的方法, 将外径 $0.61 \mathrm{~mm}$ 的 PE-10 聚乙烯管插人 大鼠 ith, 深达胸 12 至腰 1 节段. 注射用药均以生理盐水 (NS) 配制, 注射体积 $10 \mu$, 随后以 $7 \mu 1$ NS 冲洗导管内残存药物, 于 $2 \mathrm{~min}$ 内完成注射.

3. 动脉血压的描记行气管插管术后, 千左侧颈总动脉插管, 经压力换能器, 在多导生 理记录仪 (San-ei 362 型)上连续描记出 MAP.

4. 失血性休克模型的建立 (1)恒压失血性休克: 按照范镭等的方法进行 (略). （2）恒量失血性休克: 从与颈总动脉插管相接的三通管给大鼠间断性放血, 控制失血量于 $5 \mathrm{~min}$ 时 (停止失血) 达 $2.5 \mathrm{ml} / 100 \mathrm{~g}$ 体重, 即总失血量超过全血量的 $40 \%$ 最. 放血前 $10 \mathrm{~min}$, ith. 注射药物作为预处理, 观察放血开始后 $70 \mathrm{~min}$ 内 MAP 的变化.

5. ith. 滥流参照 $\mathrm{Yaksh}^{[9]}$ 的方法, 将 PE-10 自颈部沿 ith. 向尾端插人 $10 \mathrm{~cm}$, 另一 PE-10 管插人 $1 \mathrm{~cm}$ 作为收集管. 用蠕动㐮灌入经 $95 \% \mathrm{O}_{2}$ 和 $5 \% \mathrm{CO}_{2}$ 饱和的人工脑脊液, 清 入速度为 $50 \mu \mathrm{l} / \mathrm{min}$, 收集管置于冰浴中, 每 $30 \mathrm{~min}$ 收集一管, 共灌流 $2 \mathrm{~h}$, 收集的灌流液冷冻 本文 1990 年6月18日收到. 1990 年11月26日收到修改稿. 
抽干后置 $-30^{\circ} \mathrm{C}$ 冰箱保存. 放射免疫测定 (RIA). 时,每管用 $0.5 \mathrm{ml}$ 双蒸水复溶.

6. CCK-8 的放射免安测定衫I-BH-CCK-8 的放射性比活度等于 (或大于) $74 \mathrm{TBq} /$ $\mathrm{mmol}, \mathrm{CCK}-8-\mathrm{AS}$ 的效价为 1:9000, 亲和常数为 $5.5 \times 10^{11} \mathrm{~L} / \mathrm{mmol}$, 与 MEK、胃必素、 CCK-4 和无硫 CCK-8 的交叉反应均小于 $0.1 \%$, 进行 RIA 时, 反应体积为 $800 \mu 1,4^{\circ} \mathrm{C}$ 条件 下餒育 6 天后, 以二抗方法分离结合与游离部分, 用 $r$-自动免疫计数器 (LKB 9000 型, 瑞典 Pharmasia 公司产,计数率 $85 \%$ ) 测沉腚物的放射性, 该机自动绘出标准曲线并打印测定结果.

7. 统计分析文中实验数据以均值士标准误表示, 用电子计算机进行双因素方差分析 (ANOVA) 判断差异显著性.

\section{二、结 果}

1. ith. 注㣙 CCK-8 和丙谷胺对失血性休克的影响 33 只大鼠均分为三组, 恒压失 血结束时, 分别 ith 注射 NS $10 \mu 1 、 C C K-85 \mu \mathrm{g}$ 和丙谷胺 $100 \mu \mathrm{g}$, 观察注射后 $70 \mathrm{~min}$ 内 MAP 的变化. 结果表明(图 1): CCK-8 $5 \mu \mathrm{g}$ 使 MAP 的恢复明显快于 NS 对照组, 而丙谷胺 100 $\mu \mathrm{g}$ 则使 MAP 的恢复明显慢于 NS 对照组.

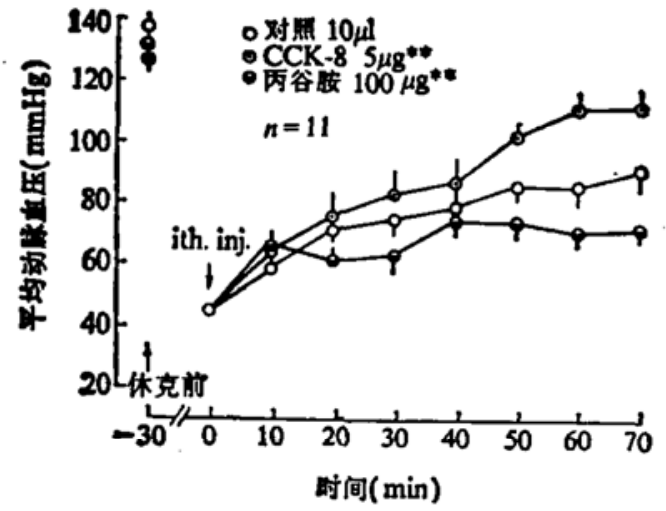

图 1 ith. 注射 CCK-8 和丙谷胺对失血珄 休克大鼠平均动脉血压的影响

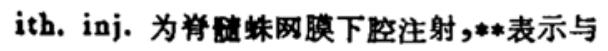
对照组相比 $p<0.01$

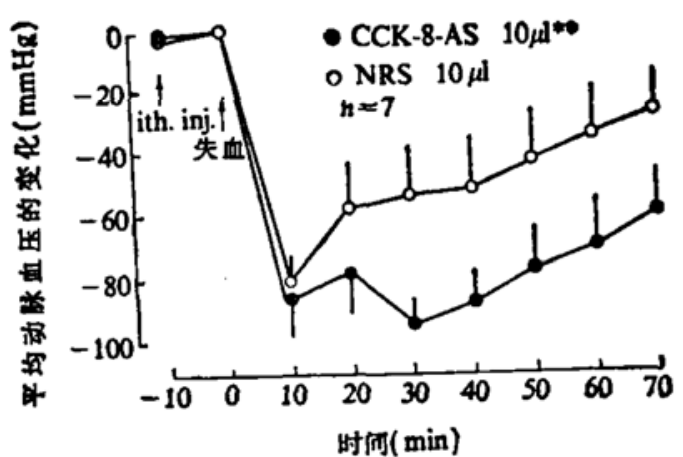

图 2 ith. 注射 CCK-8-AS 对失血性休 克大鼠平均动脉血压的影响 CCK-8-AS 为 CCK-8 抗血消, NRS 为正 常兔血清(其余图注同图 1)

2. ith. 注对 CCK-8 抗血清对失血性休克的影响

14 只大鼠均分为二组, 失血前 10 min 分别 ith. 注射正常兔血清 (NRS) 和 CCK-8 抗血清 (CCK-8-AS) 各 $10 \mu 1$, 观察恒量 失血开始后 $70 \mathrm{~min}$ 内 MAP 的变化. 图 2 表明, ith. 注射 NRS 组的 MAP 于失血后逐渐回 升, ith. 注射 CCK-8-AS 组的 MAP 从失血后 $20 \mathrm{~min}$ 开始明显 低于 NRS 组的相应值. ANOVA 的 $P$ 值小于 0.01 .

3. 失血性休克时 ith. 灌流洨中 CCK-8-ir 含年的变化恒压失血组和对照组各为 8 只大鼠, 测定 $2 \mathrm{~h}$ 灌流液中 CCK-8-ir 的含量. 结果 (图 3) 表明: (1) 对炤组第一管样品 CCK-8-ir 的含量为 $20.3 \pm 2.3 \mathrm{pg} / \mathrm{ml}$, 灌流过程中各管数值间虽有波动但无显著性差异; (2) 失血组第一管样品 CCK-8-ir 的含量为 $42.3 \pm 6.3 \mathrm{pg} / \mathrm{ml}$, 显著高于对昭组第一管样品的含 量, 也显著高于失血组其它三管样品的含量（ANOVA，p<0.01);（3)以灌流全过程而言, 失血组 CCK-8-ir 的含量显著高于对照组 (ANOVA, $p<0.01$ ). 


\section{三、讨 论}

本文结果表明:（1）给大鼠 ith. 注射 CCK-8, 可以加速失血性休克后 MAP 的恢复, 提 示中枢 CCK-8 有一定的抗休克作用; (2)失血鼠 ith. 灌流液中 CCK-8-ir 的含量明显高于对照 鼠，说明失血时内源性 CCK-8 的释放增多, 可能 有助于休克的恢复; (3)如果预先 ith. 注射 CCK8-AS 或丙谷胺使内源性 CCK-8 不能发挥作用, 则休克大鼠 MAP 的回升显著延缓, 这从反面说 明内源性 CCK-8 在失血性休克的过程中确实对 血压的降低起阻尼作用.

本实验选用了两种失血模型. 在恒压失血模 型上,观察到 CCK-8 可以加速休克的恢复,而丙 谷胺可以延缓休克的恢复 (图 1), 却未能观察到 CCK-8-AS 对休克的恢复有任何显著影响 (结果未列出). 考虑到抗血清的作用机制 ${ }^{[20]}$ 和本 实验中急性大失血刺激内源性 CCK-8释放达高峰的时间 (30 min 内, 图 3), 从方法学上讲, 失血前注射抗血清(预处理)更为合理. 可是对于恒压失血模型来说, 预处理无法排除动物代 偿反应的影响, 必然会改变实际失血总量 ${ }^{[7]}$, 因此我们改用恒量失血模型.结果 (图 2) 表明, 将 CCK-8-AS 做预处理确能延缓失血性休克后 MAP 的恢复, 与丙谷胺延缓恒压失血后 MAP 恢复的结果(图 1) 相一致.

如果中枢 CCK-8 是作为一种内源性抗阿片物质而起作用, 则应预期大失血时先有阿片 肽的释放增多,继而引发 CCK-8 的释放增多.范镭等的工作证明大鼠失血 $30 \mathrm{~min}$ 时 ith. 灌流 液中 MEK-ir 的含量升到高峰、LEK-ir 的含量于 $60 \mathrm{~min}$ 时升到高峰, 然后均呈下降趋势 [u], 而失血后中枢 CCK-8-ir 含量也是以失血 $30 \mathrm{~min}$ 内最高, 其后逐渐回降, 在时间过程上不能 反映出阿片肽与 CCK-8 两者之间有因果联系. 是否可以设想, 失血时阿片肽和 CCK-8 两 个系统同时被激活,而休克的严重程度取决于两者的相对平衡。

\section{参考文献}

[1]王肖虹等,生理学报,42(1990),3: 219-224.

[2] Faris, P. L. and Komisaruk, B. R., Science, 219(1983), 4582: 310-312.

[3] Han, J. S. et al., Pain, 27(1986), 1: 101-115.

[4]范镭、郭济生, 北京医科大学学报,21(1989),4: 264-266.

[5] Fan, L. and Han, J. S., Circ. Shock, 24(1988), 4: 423.

[6] Yaksh, T. L. and Rudy, T. A., Physiol. Behav., 17(1976), 1031-1036.

[7]范镭、䡛济生,科学通报,34(1989), 15: $1183-1185$.

[8] Collins, J. A. et al., Arch. Surg.. 99(1959), 4: 484-488.

[9] Yaksh. T. L., in Measurement of Neurotransmizter Release in vivo(Ed. Marsden, C. A.), John Wiley \& Sons Ltd., 1984, $107-124$.

[10] San, J. S., Chin. Medic J., 100(1979), 459-464. 